\title{
Medical emergency rescue in disaster: The international emergency response to the Haiyan typhoon in Philippines
}

\author{
Feng Ling ${ }^{1}$, Zhen $\mathrm{Ye}^{2, *}$, Wenwei $\mathrm{Cai}^{3}$, Ye Lu ${ }^{1}$, Shichang Xia ${ }^{1}$, Zhiping Chen ${ }^{1}$, Enfu Chen ${ }^{1}$, \\ Xiaofeng Wang ${ }^{1}$, Zhen Wang ${ }^{1}$, Huakun Lv ${ }^{1}$, Zhenyu Gong ${ }^{1, *}$ \\ ${ }^{1}$ Zhejiang Provincial Center for Disease Control and Prevention, Hangzhou, China; \\ ${ }^{2}$ Zhejiang Health and Family Planning Commission, Hangzhou, China; \\ ${ }^{3}$ The Emergency Department of Zhejiang Provincial People's Hospital, Hangzhou, China.
}

\begin{abstract}
Summary Following Typhoon Haiyan, the World Health Organization (WHO) has been supporting the Government of the Philippines in coordinating the incoming relief supplies from more than 30 international humanitarian health or 7 ganizations. During the 10 days in Abuyong, Philippines, the Chinese medical rescue team consisting of 50 experts specialized in clinical medicine and disease prevention and control action was taken including, medical treatment, environmental disinfection and health education. A total of 1,831 cases and 2,144 outpatients were treated, blood tests, B-ultrasound, electrocardiogram (ECG) and other laboratory examinations were carried out for more than 615 patients; a cumulative $\mathbf{9 0 , 0 0 0}$ square meters in external environment were disinfected, and more than $\mathbf{5 0 0}$ health education materials were handed out. Besides, measures of purifying drinking water, and rebuilding the local hospital have also been carried out. The international emergency response to the Haiyan typhoon in Philippines contributed to reconstruct the local disaster health system by the activities from international medical emergency rescue. To improve the capacity of international medical emergency rescue in disaster, the special project of foreign medical emergency rescue should be set in countries' medical emergency rescue, and disaster emergency medical rescue should be reserved as a conventional capacity.
\end{abstract}

Keywords: Medical emergency rescue, typhoon, disaster, emergency response

\section{Introduction}

The typhoon stroked the Philippines on November 8,2013 , which was the strongest in local history, causing massive destruction and 25 million people were affected. More than 5,000 people were confirmed dead, and many others had lost their homes (1). Health facilities were damaged or completely destroyed across large swathes of the affected regions, and, as a result of

\footnotetext{
*Address correspondence to:

Dr. Zhenyu Gong, Zhejiang Provincial Center for Disease Control and Prevention, 3399 Bin Sheng Road, Binjiang District, 310051 Hangzhou, China.

E-mail: zhygong@cdc.zj.cn
}

Dr. Zhen Ye, Zhejiang Health and Family Planning Commission, No.216 Qingchun Road, Hangzhou 310006, China.

E-mail: yz1668@sina.com.cn the breadth and severity of the storm, health services in the worst affected areas have ceased to exist or have been severely stretched, with medi $\neg$ cal supplies in very short supply.

Following Typhoon Haiyan, the World Health Organization (WHO) has been supporting the Government of the Philippines in coordinating the incoming relief supplies from more than 30 international humanitarian health organizations.

After the disaster, a national medical rescue team consisting of 50 experts specialized in clinical medicine and disease prevention and control was organized by Chinese government, which arrived in Abuyong, Philippines to provide medical rescue to the hospitals in Abuyong, where the local medical system has been paralyzed, and the only local hospital does not have the ability to admit patients after Haiyan. During the 10 days in Abuyong, measures of medical treatment, environmental disinfection and health education, 
purifying drinking water, and rebuilding the local hospital were carried out by our team.

\section{Medical emergency rescue measures}

Medical treatment In Abuyong, common clinical projects and public health programs were carried out by our team. According to the needs of the local health system, we set up pediatrics, obstetrics and gynecology departments, respiratory medicine, orthopedics, infection, as well as blood tests, B-ultrasound, electrocardiogram (ECG) and other ancillary departments. A sterile room was set up, the room and medical equipment were carefully disinfected using adenosine triphosphate (ATP) bioluminescence assay to detect infection during surgery - 11 times forceps and 6 times surgical scissors were sampled before surgery, which showed that the forceps surface fluorescence was four times 1 relative light unit (RLU), and the remaining test results were 0 RLU, which was in line with requirements.

During the 10 days of joint operation, we provided medical assistance to about 200 patients per day, and a total of 1,831 cases and 2,144 outpatients were treated (Figure 1). Among the diseases diagnosed, the top 5 were tuberculosis, upper respiratory tract infection, skin infection, pregnancy and scabies (Figure 2). The blood tests, B-ultrasound, ECG and other laboratory examinations were carried out for more than 615 patients; by effective disinfection of operating room and medical equipment, 26 units of surgical operations were conducted successfully. Because of the large amount of patients, drugs we brought were not enough. So after consulting the embassy staff, many common drugs were bought, such as cloxacillin, erythromycin, paracetamol, amoxicillin, paracetamol, cotrimoxazole, and povidone iodine, mediplast, needles.

Besides, we also gave some guidance to the hospitals in Abuyong on the aspects of disinfection of medical pre-screening of clinics, obstetrics and the operating room, especially disinfecting support for the first medical surgery, disinfection testing,

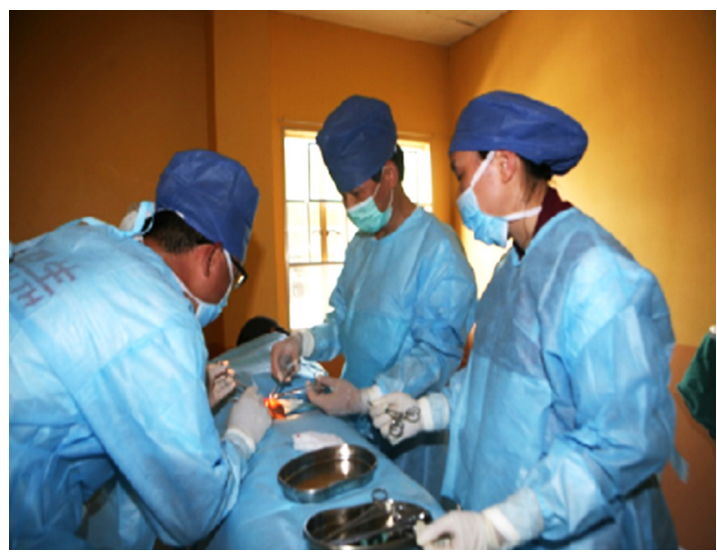

Figure 1. The first case of hip tumor resection carried out by Chinese medical rescue team. and sterilization of surgical instruments to prevent nosocomial infections.

Environmental disinfection and health education Microbiological indicators on surroundings were monitored by ATP bioluminescence assay of medical equipment and surgical instruments, while the quality of water was detected using a chlorine analyzer (H293711, Italy HANA) and density of mosquitoes was measured by visual method in the external environment (Figure 3 ). With one effervescent tablet (500mg/tablet) plus $1 \mathrm{~L}$ water to allocate $500 \mathrm{mg} / \mathrm{L}$ of disinfectant for residual spraying, and treatment tablets and medical instruments with wipe disinfection; and added chlorine to water which had inadequate residual chlorine by detection; the external environment insecticide used was deltamethrin (content of $2.5 \%$, EC, Bayer UK). A cumulative 90,000 square meters in the external environment were disinfected, besides, more than 500 health education materials were handed out, which effectively prevented

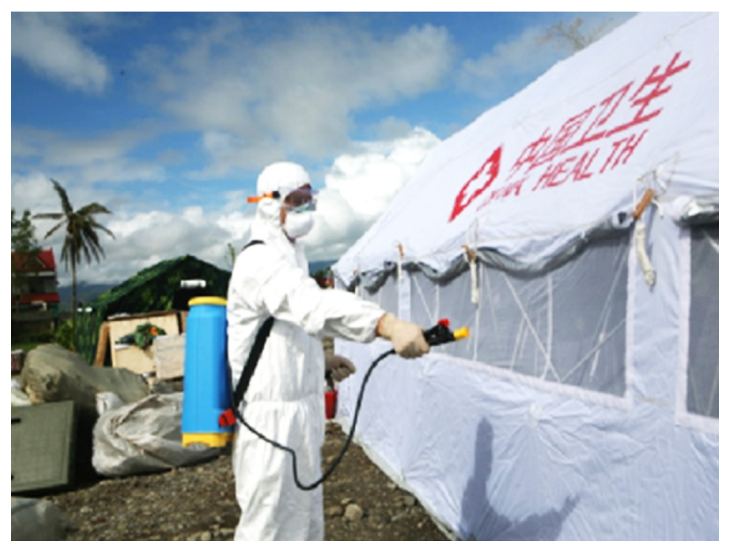

Figure 2. The team member disinfected and used insecticide around the camp.

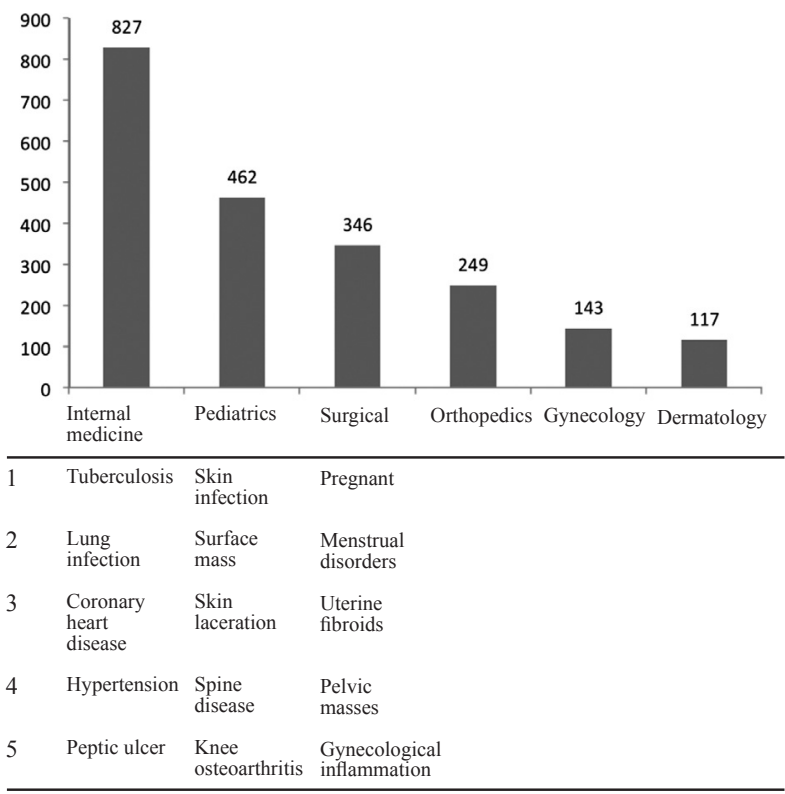

Figure 3. Classification of patients for the Chinese emergency rescue team diagnosis and treatment in Abuyong. 
the occurrence of infectious diseases after Haiyan.

Purifying drinking water Because of Haiyan, running water, electricity, and communication were all interrupted, and there was a lack of enough drinking water. Thus, we immediately communicated with the local residents after arrival, and found that drinking water was mainly from wells and rivers without special disinfection. In addition, it was rainy season at that time when the amount of rain was three times more than normal per day. To ensure enough clean and healthy drinking water, chlorine was added and timely detected in water. The turbidity of untreated water was 3.62 , with total chlorine zero. After estimation of the size and depth of the well, 30 disinfection tablets were put in, then we purified the water by polypropylene cotton, activated carbon and ultra filtration; after these measures, the turbidity of water declined to 0.2 . As local residents and us shared the same well and did not like the sense of chlorine, we controlled the total chlorine to less than 0.2. Until December 1st, luckily local residents did not object drinking water with chlorine. When local residents got used to the sense, we increased the amount of disinfection tablets to 0.3 in tap water.

Rebuilding the the local hospital Because of the impact of the typhoon, the only one hospital - Abuyong Aeneral Hospital in Abuyong was on the verge of paralysis. The diagnostic equipment in hospital cannot work because of lack of power, which also brought great difficulty for rebuilding the local hospital system. On the recommendation of the local government, we took over the local hospitals to gradually restore their treatment capacity. We helped and trained them to carry out medical work after the disaster, when they had no drugs, no instruments and irregular medical materials, and also when we evacuated from Abuyong, we donated lots of prerequisite materials and medical supplies, such as generators, medical monitors, ECG, clinical urinary analyzers, infrared thermometers, oxygen generators, suction equipment, glucometers and operation lamps, disposable surgical packages, disposable surgical gowns, infusion apparatus, N95 masks, disposable latex gloves and a large quantity of pharmaceuticals.

\section{Discussion}

From SARS (2003) to Wenchuan earthquake (2008), from Yushu earthquake (2010) to Ludian earthquake (2014), from the supporting medical team fighting against Haiyan typhoon in Philippines in 2013 and that for Ebola in Africa in 2014, the Chinese rescue teams against public disasters have made great progress and played a more important role, not only for national medical emergency rescue, but also for international public health emergency rescue.

This international medical aid to the Haiyan typhoon in Philippines gained much support from the
Chinese Government and many departments, including the Embassy, the Civil Aviation Bureau, the Customs Head Office, and General Administration of Quality Inspection. They facilitated material preparation, charter transportation and so on. Specific workers are assigned to accompany the medical team, help contact our own country and communicate with the local people. With their help, our medical team members could communicate fluently with the local government, local people and local army, and then participate in disaster relief activities together, which have improved efficiency and effectiveness of relief activities, as well as safety of the medical team.

Our experience suggests that the WHO guidelines (2) are sound and appropriate when there is a total collapse of the local medical infrastructure. When the local medical system was in paralysis or semi paralyzed, the cooperation between the rescue teams from other countries and the local medical institutes can help gain confidence of the local people to the foreign rescue teams on one hand, and on the other hand, the cooperation could contribute to the recovery of the local medical system.

In international rescue especially in typhoon rescue, the water would always be polluted. When self-brought water could not satisfy need during the long period of rescuing, turbidity meter and total chlorine meter could be used to test the total particulate matter and the total chlorine quickly. It would be easy to satisfy the need in a disaster area, if we filter the local water with PP, activated carbon and gradocol membranes.

The devastation in the Philippines is only one of many recent examples of destruction from weather phenomena (3), a special project of international medical emergency rescue should be set in countries' medical emergency rescue, and disaster emergency medical rescue should be reserved as a conventional capacity. Unfortunately, lopsided development of medicine is generally worldwide. Some countries with a low-level medical system are always subjected to natural disasters. They are not always capable of carrying out rescues. In such situations, border countries or even some developed countries should carry out international medical emergency rescue. With the development of international communication, such foreign medical emergency rescue would increase. Thus, medical emergency relief teams with miniaturized and portable equipment, with a capability of distant transportation and with standard logistic support should be built. When disasters happen, one country should bear the social and public opinion on long - term development with border countries in mind and act according to its ability.

We should also point out that there are still some shortages of Chinese medical rescue teams which have to improve the capacity of its own for all the 50 medical rescue staff - there are only 6 professional public health experts $(6 / 50)$, who play an important role 
in guaranteeing the public health of a rescue camping site (safety of drinking water and food, density control of vectors, etc.). Due to limited human resources and the local special environment, we could not be able to launch large-scale help in the rescue area, including case searching, safety of drinking water and food and other public health work.

Of course, we have shortages in collected data, which were produced during the process of rescue, such as, we do not have the density data of mosquitos before insecticide use, we also did not calculate the amount of disinfectant to use every day for living water disinfection.

In conclusion, the international emergency response to the Haiyan typhoon in Philippines contributed to reconstruct the local disaster health system by measures from international medical emergency rescue. To improve the capacity of international medical emergency rescue in disasters, the special project of international medical emergency rescue should be set in countries' medical emergency rescue, and disaster emergency medical rescue should be reserved as a conventional capacity.

\section{References}

1. WHO coordinates health relief after typhoon Haiyan. Bull World Health Organ 2013; 91:900-901.

2. WHO-PAHO guidelines for the use of foreign field hospitals in the aftermath of sudden-impact disasters. Washington, DC: Pan American Health Organization, 2003 (http://www.who.int/hac/techguidance/pht/ FieldHospitalsFolleto.pdf). (accessed December 15, 2014)

3. Cranmer HH, Biddinger PD. Typhoon Haiyan and the professionalization of disaster response. N Engl J Med. 2014; 370:1185-1187.

(Received November 4, 2014; Revised December 21, 2014; Accepted December 23, 2014) 